\title{
DESIGN DE WEARABLES PARA USO ESPORTIVO: SENSORES INERCIAIS APLICADOS À NATAÇÃO EM PISCINAS
}

\author{
Gabriela Trindade Perry \\ Universidade Federal do Rio Grande do Sul \\ gabriela.perry@ufrgs.br \\ Artur Becker \\ Universidade Federal do Rio Grande do Sul \\ arturbecker94@gmail.com \\ Gabriel Marins \\ Universidade Federal do Rio Grande do Sul \\ gmcmarins@gmail.com
}

Resumo: Este trabalho refere-se ao projeto e construção de wearables de baixo custo com foco na natação em piscinas, utilizando sensores inerciais para captura de dados com fins científicos. De forma ampla, os resultados deste projeto podem estender-se para diversas modalidades esportivas, com aplicações acadêmicas ou de ensino, tanto para atletas de alto rendimento como atletas amadores. Dispositivos desta natureza facilitam muito a coleta de dados "na borda da piscina", que atualmente são obtidos a muito custo. Estes dados são variáveis importantes na compreensão do rendimento do atleta, pois provavelmente se correlacionam com indicadores fisiológicos e biomecânicos. O esforço no sentido de desenvolver tais dispositivos tem se intensificado desde 2010, em função da diminuição do custo de componentes eletrônicos utilizados em tais dispositivos, e do desenvolvimento da tecnologia destes componentes.

Palavras-chave: Wearables, sensores inerciais, natação. 


\section{INTRODUÇÃO}

A evolução no desempenho de atletas depende de diversos fatores, subjetivos e objetivos. Prazer em praticar o esporte, disposição e disciplina são fatores subjetivos, que podem até certo ponto ser modulados conforme a vontade do atleta. Aptidão é um fator objetivo, de caráter biológico, que pode ser o condicionante para o sucesso do praticante de alto desempenho, mas que dificilmente irá impedir a prática a nível amador ou recreativo. Condicionamento físico e técnica também são fatores objetivos, que serão determinantes na história do atleta de qualquer nível. Afora fatores biológicos, todos os demais podem ser desenvolvidos.

No caso deste projeto o objetivo principal está relacionado com a compreensão de fatores biomecânicos, que são importantes para o sucesso em certas modalidades e absolutamente essenciais no caso da natação. Atletas que têm domínio da técnica do nado são mais velozes e deslocam-se com menor gasto energético, sendo muito mais eficientes que um atleta com baixo domínio da técnica. Estes fatores biomecânicos serão modelados em variáveis capturadas pelos wearables desenvolvidos neste projeto. As das variáveis de interesse neste projeto dizem respeito à rotação e à velocidade, em pontos representativos do corpo do atleta.

Consideramos que dispositivos que capturem estes dados em tempo real possam ser de grande valor para a pesquisa científica porque a técnica do nado é de difícil observação, tanto em função do meio aquático (o parecer do observador sobre o desempenho do atleta sofre com o fenômeno da paralaxe) como da sutileza, coordenação e velocidade dos movimentos. Há diversos desafios nesta coleta, tanto em relação ao processo de captura como em relação à interferência do próprio meio aquático. Atualmente (em 2016) estes dados são capturados em um ambiente laboratorial e não familiar, o que pode acarretar efeitos psicológicos e fisiológicos no atleta. Assim, é preferível a utilização de dispositivos de medição e análise in situ, nas instalações de treinamento ou competição utilizadas pelo atleta (JAMES, 2008). Estes dados provavelmente estão correlacionados com o desempenho do atleta, e por isso sua obtenção é essencial (CALLAWAY, COBB, JONES, 2009).

\section{WEARABLES PARA NATAÇÃO}

Grande parte dos wearables para esporte e saúde consiste de sensores montados no corpo, que coletam dados que são armazenados em cartões de memória ou transmitidos via wireless. Dependendo da aplicação estes sensores precisam ser posicionados em partes específicas do corpo para obter informações, tais como medidores de pulsação. Para tornar o sistema menos obstrutivo, é possível integrá-lo à roupa. No contexto de saúde e esporte, os sensores mais comumente utilizados são os fisiológicos e os biomecânicos. Sensores fisiológicos leem sinais como frequência cardíaca, de respiração, e temperatura. Sensores biomecânicos medem parâmetros de movimento de segmentos do corpo, os mais comuns sendo os sensores de navegação inercial (SCILINGO, et al, 2011).

No caso da natação, a necessidade de ser a prova d'água dificulta o desenvolvimento de dispositivos, de forma que mesmo tocadores de música se tornam caros e de uso incômodo. A Figura 1 mostra alguns exemplos de wearables, selecionados de um site especializado (SUNG, 2015) e de um fabricante de wearables (XMetric, sem ano). 


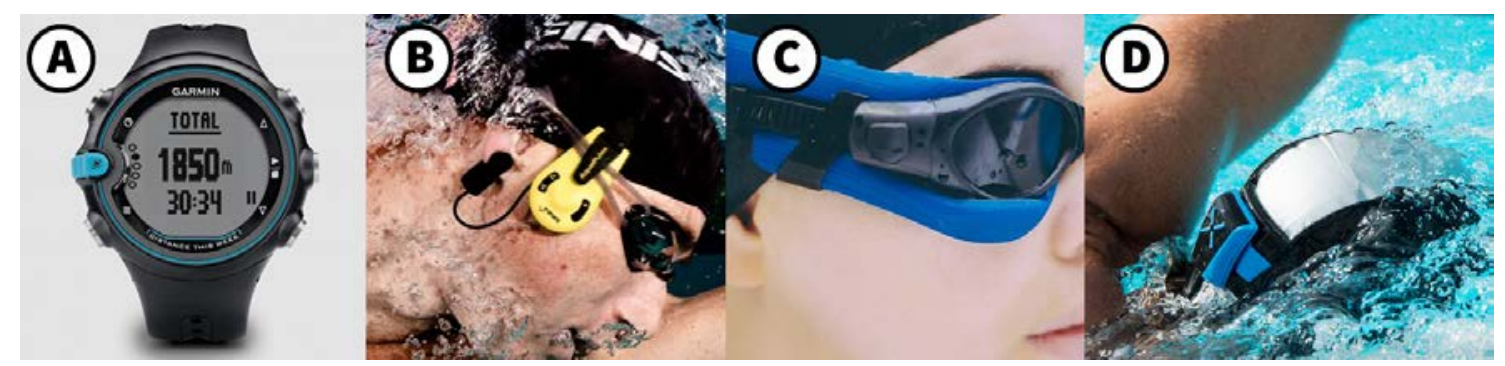

Figura 1 - Exemplos de wearables para natação.

A Figura $1 \mathrm{~A}$ mostra um relógio da marca Garmin, que traz, além de um cronômetro, um contador de voltas e um GPS. Os dados podem ser enviados ao celular, que mostram dados de desempenho do nadador. Na Figura $1 \mathrm{~B}$ e $1 \mathrm{C}$ vemos dispositivos que podem ser acoplados ao lóbulo auricular e aos óculos, e que mostram dados da frequência cardíaca. Em 1B vemos o produto Finis Acqua Pulse, que envia um estímulo sonoro. Em 1C o Instabeat, que projeta luzes nos óculos de acordo com o perfil de frequência (queima de gordura, condicionamento aeróbio e treinamento cardiorespiratório). Em 1D mostramos o produto mais completo encontrado, um dispositivo que é fixado na touca do atleta e que captura todos os dados dos dispositivos anteriores de forma não obstrusiva. Além disso, podem-se descarregar os dados em um software que os interpreta e faz uma análise do progresso do treinamento. 0 problema é que todos estes equipamentos são caros, e, fora o relógio Garmin, sequer estão disponíveis no Brasil.

\section{MÉTODOS PARA COLETA DE DADOS EM NADADORES}

Os métodos para coleta de dados que serão mostrados neste artigo são aplicados à pesquisa em natação, que costuma restringir a amostra a nadadores competitivos - uma parcela muito, mas muito pequena da população de nadadores. 0 motivo é que ao restringir as pesquisas a nadadores que estão no limiar do desempenho humano torna-se mais simples identificar o efeito de manipulações experimentais - como em variações em rotinas de treinamento, por exemplo - pois as variações de desempenho entre os indivíduos são mínimas.

\subsection{Videogrametria}

Atualmente a captura de dados sobre posicionamento, velocidade e rotação em nadadores são feitas através da análise de vídeo (videogrametria) - e este método torna extremamente restrita a sua aplicação (Magalhães et al 2014). Além dos preparativos para a coleta serem demorados, a análise dos dados exige um esforço computacional muito grande e impõe uma alta demanda cognitiva sobre o analista (que deve ser, necessariamente, um especialista neste método de coleta). Neste cenário, o uso desta técnica está restrito a instituições científicas e centros esportivos de elite. Outras limitações da coleta de dados por vídeo incluem a limitação da amostra (devido às limitações técnicas, o material coletado em vídeo costuma representar apenas um ciclo de braçada do nadador, em um trecho específico da piscina), a paralaxe do meio aquático e artefatos na imagem capturada (bolhas, turbulência, ocultamento de partes do corpo). Estes fatores contribuem para que 
treinadores percebam a videogrametria como tendo utilidade prática limitada, apesar dos mesmos reconhecerem a importância da análise quantitativa de desempenho (Mooney et al 2015). A Figura 2 mostra o procedimento de preparação de um atleta para o ensaio.
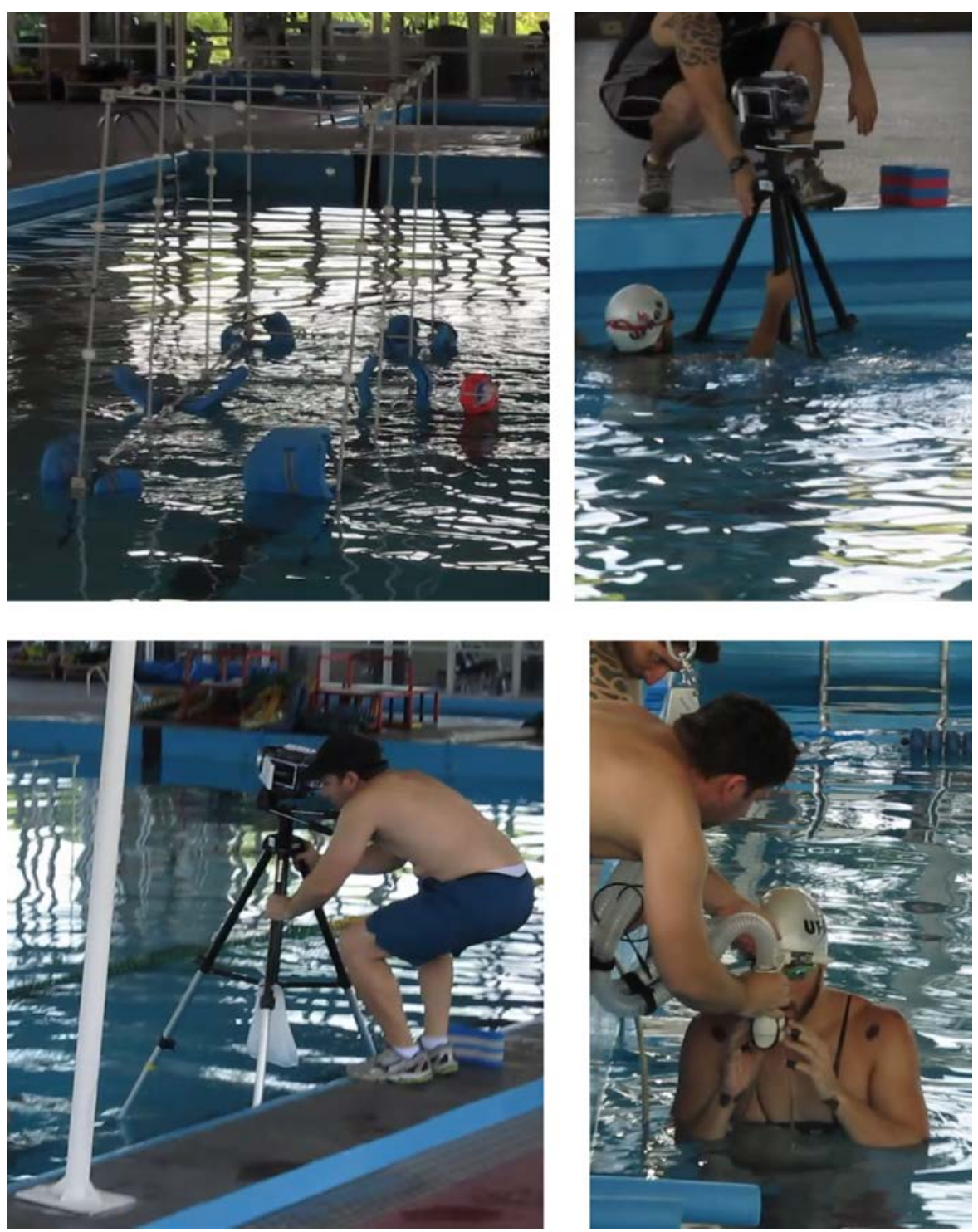

Figura 2 - Preparação para coleta de dados usando videogrametria.

Em contraponto à videogrametria, há um crescente corpo de estudos versando sobre a utilização de sistemas de navegação inerciais (INS - inertial navigation system) como alternativa para a coleta de dados de desempenho.

\subsection{Sistemas de navegação inerciais}

Sistemas de navegação inerciais (acelerômetro, giroscópio e bússola) são utilizados para captar informações-chave sobre o desempenho do atleta, como velocidade, contagem de ciclos, simetria do nado, entre outras. Além disso, desenvolvimentos recentes da tecnologia dos sensores inerciais viabilizam a criação de sistemas de baixo custo e de pequenas dimensões, que podem ser fixados diretamente 
no corpo de atletas, embora, principalmente no caso de atletas de alto desempenho, ainda careçam investigações sobre o efeito do arrasto gerado pelo sistema (Magalhães et al 2014).

Comparados à videogrametria, sensores inerciais têm o potencial de ter um custo muito menor de aquisição e de operação (dispensa um sistema de multi-câmeras e o trabalho de técnicos para captação manual dos dados), além de poderem ser aplicados durante toda a distância percorrida pela atleta, apresentando dados de performance mais representativos e possibilitando a análise do efeito da fadiga sobre a técnica de nado, além de possibilitar que múltiplos atletas possam ser analisados ao mesmo tempo, na mesma piscina. Esses fatores levaram pesquisadores a propor que o uso de sensores inerciais poderia ser vantajoso em relação à videogrametria tradicional (CALLAWAY, COBB, JONES, 2009).

Alguns autores vêm desenvolvendo, com sucesso, sistemas para medição de diferentes aspectos do nado utilizando sensores inerciais. Dadashi (2012), desenvolveu algoritmos para a obtenção do perfil de velocidade de um atleta durante um tiro e o perfil de velocidade intra-cíclica para cada braçada. Os dados foram captados por um sensor inercial tri-axial fixado na lombar do atleta, e computados posteriormente informando-se a dimensão da piscina e o tempo cronometrado manualmente da prova, para contornar os erros de integração do sinal. Os ciclos de braçadas eram reconhecidos através do rolamento do corpo do atleta, graças à captação de aceleração médio-lateral, de forma similar à utilizada por Stamm (2015), onde foi utilizado um algoritmo de zero-crossing para detectar o número de braçadas e analisado o sinal da aceleração no eixo médio-lateral para verificar de qual lado é cada braçada (na configuração do autor, a aceleração da gravidade no eixo médio-lateral era positiva para braçadas com o lado direito e negativa para braçadas com o lado esquerdo). Em outro estudo, Stamm (2012), também utilizando um acelerômetro fixado na lombar do atleta, mediu seu perfil de velocidade comparativamente ao obtido por um sistema de amarra (composto por um rolo de cabo com uma extremidade acoplada à vestimenta do atleta e a outra na borda da piscina, com um sensor óptico que mensura quanto o cabo está se desenrolando por segundo), normalizando a velocidade obtida da integração do sinal do acelerômetro para a velocidade média obtida sabendo-se o comprimento da piscina e o tempo da prova manualmente cronometrado. A análise das variações de aceleração nos três eixos permitiu aos pesquisadores discernir 5 pontos chave do tiro: início do tiro, fim do pushoff, começo do nado, fim do nado e fim do tiro.

Também utilizando sensores inerciais, Le Sage (2010) buscou criar um sistema de análise de desempenho holístico, com feedback em tempo real para múltiplos atletas. O sistema - focado em contagem de ciclos e duração dos mesmos - é composto por uma rede de sensores sem fio (WSN), uma câmera debaixo d'água e outra de altavelocidade do lado de fora da piscina, uma placa de força adaptada ao bloco de largada, marcadores de LED em pontos chave no corpo do atleta, um gatilho de áudio e laptops, além de fones à prova d'água para comunicação em tempo real do treinador com o atleta. Cada nó da rede é um sensor de inércia tri-axial, fixado na região da lombar de cada atleta, o que permite a análise de desempenho de diversos atletas simultaneamente durante um treino. A autora conclui apontando que, utilizando-se métodos manuais para obtenção dos dados (i.e contagem de voltas, contagem de braçadas e sua duração) através do vídeo ou da análise das curvas obtidas, são 
necessários 45 minutos para cada 100 metros de nado, ou 45 horas para uma sessão de duas horas de um nadador de elite. Os mesmo dados foram obtidos em tempo real através dos algoritmos de detecção desenvolvidos no estudo.

Todos estes autores reconhecem a questão do erro oriundo da integração de dados de um único acelerômetro para obtenção da velocidade, mas através de diversos métodos de filtragem e análise dos algoritmos, o erro é controlado para a duração de movimento de interesse. Le Sage (2009), por exemplo, propôs o uso do Filtro de Kalman com identificação de máximos e mínimos de aceleração para identificação das voltas na piscina, o que possibilita que o filtro seja "resetado" a cada volta e a posição do atleta seja atualizada automaticamente adicionando-se o comprimento, conhecido previamente, da piscina. Stamm (2012) utilizou um acelerômetro em repouso para medir o erro de integração para o intervalo de tempo do tiro (considerado de no máximo 40 segundos) como $0,08 \mathrm{~m} / \mathrm{s}^{-1} 1$, ou $0,002 \mathrm{~m} / \mathrm{s}^{-1} 1$ por segundo integrado, e conseguiu obter resultados similares de velocidade do atleta à medida pelo sistema de amarras.

\subsection{Aspectos técnicos de sensores inerciais}

Sensores inerciais de navegação são sensores cujas medições são usadas para rastrear a posição e orientação de um objeto em relação a um ponto de partida, orientação e velocidade conhecidos. Unidades de medição inercial (IMU - Inertial Measurement Unity) tipicamente contêm três giroscópios e acelerômetros ortogonais, medindo velocidade angular e aceleração linear, respectivamente. Há modelos que acrescentam uma bússola de três eixos, oferecendo um sistema com nove eixos. Mediante o processamento de sinais a partir destes dispositivos seria possível rastrear a posição e orientação de um objeto.

A navegação por inércia é utilizada numa vasta gama de aplicações, incluindo a navegação de aeronaves, submarinos e navios, estabilização de imagens, jogos e diversas modalidades de interação em softwares. Recentes avanços na construção de MEMS (Micro-Electro-Mechanical Systems) tornaram possível a fabricação de sistemas pequenos, leves e baratos. Estes avanços ampliaram a gama de aplicações possíveis para incluir áreas como o estudo do movimento humano e animal.

Atualmente há diversos modelos de INS disponíveis no mercado, sendo que as diferenças estão no custo, na precisão, na confiabilidade e na disponibilidade no mercado brasileiro.

A Tabela 1 mostra alguns modelos, avaliados (pelos autores) segundo critérios que julgamos relevantes para o uso do componente neste trabalho. 
Tabela 1 - Modelos e características de alguns INS.

\begin{tabular}{|l|c|c|c|c|c|}
\hline & $\begin{array}{l}\text { Adafruit 9- } \\
\text { DOF } \\
\text { Accel/Mag/ } \\
\text { Gyro }\end{array}$ & $\begin{array}{l}\text { MPU 6050 } \\
\text { (InvenSense) }\end{array}$ & $\begin{array}{l}\text { MPU 9250 } \\
\text { (InvenSense) }\end{array}$ & $\begin{array}{l}\text { X6-2mini } \\
\text { (Gulf Coast } \\
\text { Data } \\
\text { Concepts)*** }\end{array}$ & $\begin{array}{l}\text { uINS-2 } \\
\text { (Infusionsoft) }\end{array}$ \\
\hline Custo em Reais & $\mathrm{R} \$ 76,00 *$ & $\mathrm{R} \$ 16,00$ & $\mathrm{R} \$ 24,00 *$ & $\mathrm{R} \$ 540,00 *$ & $\mathrm{R} \$ 3.600,00 *$ \\
\hline Disponível no Brasil & $\mathrm{Não}$ & $\mathrm{Sim}$ & $\mathrm{Não}$ & $\mathrm{Não}$ & $\mathrm{Não}$ \\
\hline $\begin{array}{l}\text { Estimativa de custo } \\
\text { total, considerando } \\
\text { impostos e custo de } \\
\text { frete, para 1 peça** }\end{array}$ & $\mathrm{R} \$ 460,00$ & $\mathrm{R} \$ 0,00$ & $\mathrm{R} \$ 335,64$ & $\mathrm{R} \$ 1.300,00$ & $\mathrm{R} \$ 5.600,00$ \\
\hline Quantidade de eixos & 9 & 6 & 9 & 6 & 9 \\
\hline $\begin{array}{l}\text { DMP / Sensor Fusion } \\
\text { documentado }\end{array}$ & Sim & Sim & Sim & Não & Sim \\
\hline $\begin{array}{l}\text { Chip integrado a um } \\
\text { GPS }\end{array}$ & Não & Não & Não & Não & Sim \\
\hline
\end{tabular}

* Preço em anunciado em Dólar Americano, convertido em Real Brasileiro, usando a relação \$1,00 = $R \$ 3,53$ (dia 19 de abril de 2016). Este valor não representa o preço final, pois devem ser somados custos com importação e imposto.

** Calculado através do site http://www.tributado.net/ e considerando frete de $\$ 40,00$ (UPS).

*** O X6 não é um chip, e sim um produto completo, que está pronto para ser usado.

A dificuldade em obter os dados a partir destes INS fica evidente na página sobre o MPU 6050 (o único disponível no Brasil em 2016) no web site do Arduíno : Reading raw values is easy, the rest is not. Os dados do acelerômetro contém ruído quando a leitura é feita em intervalos curtos, e os dados giroscópio derivam em escalas de tempo longas, de modo que é preciso um filtro para estes dados. Além disso, os dados separados não trazem a informação completa sobre o estado do sistema, como David Sachs (2010) afirma. A Figura 3 mostra a saída de um acelerômetro e de um giroscópio, e nela pode-se ver que ambos os sinais têm ruído. Ao longo de sua apresentação, Sachs mostra como ambos componentes apresentam comportamentos não esperados em determinadas condições (quando se faz um movimento muito rápido o acelerômetro mostra picos de aceleração inexistentes, e quando se para de movimentar o giroscópio estaciona em $0,0,0$ ).

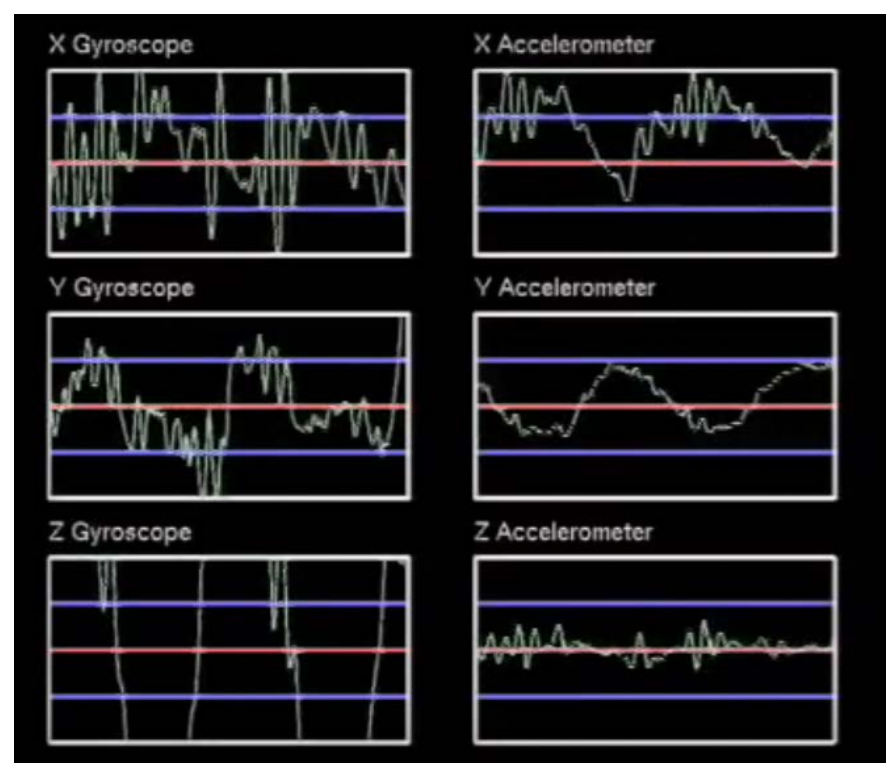

Figura 3 - Leituras de um acelerômetro e de um giroscópio, mostradas na palestra de Sachs (2010). 
No entanto, o MPU-6050 contém um processador digital de movimento (DMP) que pode integrar estes dados no chip IMU. Embora os detalhes exatos de como o DMP faz estes cálculos não serem públicos, o DMP ainda é útil porque, presumivelmente, a fabricante (Invensense) tem um algoritmo de fusão muito bom, e porque os cálculos são feitos no chip MPU-6050, liberando energia do processador do Arduíno, que tem um potencial consideravelmente menor.

Estas limitações foram contornadas com a publicação de uma biblioteca desenvolvida por Jeff Rowberg e disponibilizada de forma aberta no Github. Segundo o site i2CDevLib, fonte indicada pelo autor da biblioteca como url do projeto,

Devido à falta de uma boa documentação publicamente disponível sobre o funcionamento interno deste dispositivo [MPU 6050], todas as informações relacionadas com a DMP têm sido obtidas através de engenharia reversa, a partir de análise de sinais $12 \mathrm{C}$ correlacionados com o software Embedded MotionApps, fornecido pelo empresa. Este esforço de descoberta está incompleto e em curso, e deve resultar em uma biblioteca que suporte completamente o DMP. Atualmente, o código fonte disponível só irá fornecer a configuração básica do dispositivo e leituras de aceleração / giroscópio (o que é certamente útil, mas que falha ao entregar o principal ponto de venda da série MPU-6000, que é a capacidade de processamento de movimento pelo dispositivo)" (ROWBERG, sem ano)

Este texto de Rowberg não possui data. Porém no Github pode-se ver que a última atualização da biblioteca MPU6050 foi em janeiro de 2016 - o que indica que o desenvolvimento dela está ativo.

A insistência em usar o MPU 6050, apesar de todos estes problemas, é que ele é o único sensor de movimento com 6 eixos e DMP comercializado no Brasil, em 2016. Há outros sensores no mercado, como se pode ver na Tabela 1, porém o custo de importá-los é muito alto. Mesmo que este projeto conte, futuramente, com recursos financeiros para aquisição de equipamentos ou mesmo com a facilitação de importação para equipamentos usados em pesquisas, este custo inviabiliza nosso objetivo de desenvolver um wearable que tenha potencial de comercialização, como um produto. Afinal, o custo total de um wearable que contem um INS de $\mathrm{R} \$ 16,00$ será bem menor do que um que contenha um INS de $\mathrm{R} \$ 5.600,00$. Além disso, como este projeto tem por objetivo o desenvolvimento de um wearable para uso em pesquisa, é preciso que tenhamos acesso ao processo de captura e tratamento dos dados (isso excluiria o X6). Não é necessário que todos os códigos sejam desenvolvidos pela equipe, mas sim que os códigos usados sejam abertos, de forma que possamos analisar suas limitações e fazer alterações se for necessário.

\section{DESENVOLVIMENTO}

A extração dos dados da rotação do MPU 6050 é feita diretamente com os dados do DMP, de forma que esta variável não chega a ser um obstáculo importante. 0 grande problema é a extração da velocidade e da posição. Woodman (2007) ilustra como estes dados são obtidos (Figura 4). Woodman (2007) aponta limitações em relação à obtenção de dados destes sensores: necessidade de filtragem do sinal em função de ruído produzido pelo equipamento (provavelmente se possa usar um low pass filter); necessidade de correções do sinal (correção dos valores considerando a gravidade da Terra, que provavelmente não é desejada na maior parte das aplicações); acúmulo de erros devido ao cálculo da velocidade e da posição em função da 
aceleração - que podem introduzir erros intoleráveis em menos de 10 segundos de leitura (Gilbert et al, 2010).

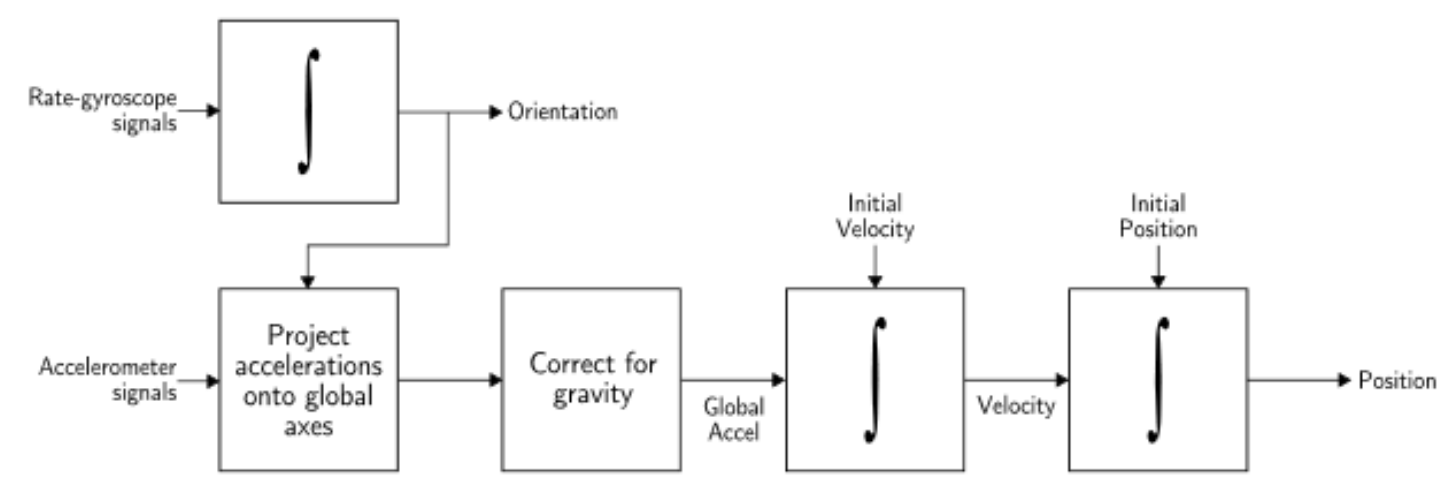

Figura 4 - Algoritmo para extração da posição e da velocidade.

A primeira e a segunda integrações da aceleração resultam respectivamente na velocidade e na posição. Como se partirá de uma posição e de uma velocidade conhecidas (em t0 a velocidade e a posição serão iguais a 0 ), estes valores serão usados como a constante da integração. Todavia, segundo Sachs (2010), esse processo pode causar um erro de 8,5 metros em 1 segundo.

O outro é o erro acumulado nas sucessivas etapas da integração. Seifert \& Camacho (2007) sugerem usar o método de aproximação de primeira ordem para fazer a integração, de modo que o erro seja reduzido. Este é o problema que parece, neste momento da pesquisa, ser o mais sério, pois não poderia ser contornado completamente. A pesquisa que fizemos aponta que a melhor solução é utilizar o Filtro de Kalman, um algoritmo que estima o estado $t n+1$ das variáveis de posição e velocidade, através do nível de confiança das medições anteriores de cada sensor.

Gilbert et al (2010), tomando uma rota diferente, testaram um integrador analógico, que teve resultados satisfatórios em uma leitura de mais de 10 segundos.

\section{CONCLUSÃO}

No presente estágio do trabalho não sabemos se conseguiremos usar apenas o MPU 6050 para capturar os dados da velocidade, por isso consideramos usar, nesta ordem: um emissor de rádio frequência e um shield GPS.

Não é requisito deste projeto a análise dos dados em tempo real. Sendo assim, os dados podem ser armazenados (local ou remotamente) e analisados a posteriori, em um software como o Matlab, R ou SPSS. Isso liberaria o Arduíno de processar os dados, o que diminui a complexidade do projeto computacional.

No mês de maio de 2016 montamos um "protótipo seco" extremamente simples, com 3 protoboards e 3 Arduínos. Este protótipo tinha como único objetivo avaliar o erro na integração da aceleração em distância pequenas. Duas protoboards continham um Arduíno cada, e, ligadas a cada um deles, havia um sensor de luz LDR (light dependent resistor). Estes sensores enviavam para a porta serial o instante em que detectavam uma variação na iluminação ambiente. Cada uma destas protoboards estava ligada a um computador, separados por cerca de 3 metros. A terceira protoboard continha um Arduíno e um MPU6050 e um botão ligados a ele, sendo que todo o sistema era 
alimentado por uma bateria, de forma que não era necessário estar ligado a um computador. O método era de avaliação foi muito rudimentar: duas pessoas iniciavam a contagem do tempo nos computadores através do envio de um caractere para a porta serial (ambas deveriam apertar qualquer tecla do teclado ao mesmo tempo) e uma terceira, portando o sistema móvel com o MPU6050, acionava o botão. Esta terceira pessoa deveria então interromper o sinal do sensor LDR nos dois terminais. A forma de comparação era confrontar a velocidade calculada pelos dados enviados pelo MPU (integrados uma vez para encontrar a velocidade através da aceleração) com a velocidade calculada pela diferença do tempo do disparo do sensor LDR nos dois terminais. Como os três sistemas não foram acionados exatamente no mesmo momento, há um erro - que não sabemos estimar. Porém este teste foi mais do que suficiente para vermos que os dados parecem estar numa escala razoável - com um erro muito menor do que o esperado pelas análises de videogrametria.

Sendo assim, nossa conclusão é que o projeto é promissor, e que estamos no caminho certo.

\section{REFERÊNCIAS}

CALLAWAY, Andrew J; COBB, Jon E; JONES, Ian. A Comparison of Video and Accelerometer Based Approaches Applied to Performance Monitoring in Swimming. International Journal of Sports Science \& Coaching 4(1):139-153. 2009.

DADASHI, F. et al., 2012. Front-Crawl Instantaneous Velocity Estimation Using a Wearable Inertial Measurement Unit. Sensors, 12(10), p.12927-12939.

DAVEY, N. P.; JAMES, D.A. Swimming stroke analysis using multiple accelerometer devices and tethered systems. The Impact of Technology on Sport II. Edited by A . Subic , F . K . Fuss, and S . Ujihashi Taylor \& Francis. 2008.

FERREIRA, R.L., 2007. Políticas para o Esporte de Alto Rendimento - Estudo comparativo de alguns sistemas esportivos nacionais visando um contributo para o Brasil. XV Congresso Brasileiro de Ciências do Esporte, (1), p.1-11.

GILBERT, H.B., CELIK, O. \& O'MALLEY, M.K., 2010. Long-term double integration of acceleration for position sensing and frequency domain system identification. In IEEE/ASME International Conference on Advanced Intelligent Mechatronics, AIM. pp. 453-458.

LE SAGE, T. et al. 2009. Kalman filter design for application to an INS analysing swimmer performance. 18th European Signal Processing Conference (EUSIPCO). Aalborg, Denmark, August 23-27. p.1723-1727.

LE SAGE, T. et al., 2010. A component based integrated system for signal processing of swimming performance. Proceedings of the 2010 International Conference on Signal Processing and Multimedia Applications (SIGMAP), p.73-79

MAGALHAES, F.A.D. et al., 2014. Wearable inertial sensors in swimming motion analysis: a systematic review. Journal of sports sciences, 2015, p.1-14.

MOONEY, R. et al., 2015. Inertial sensor technology for elite swimming performance analysis: a systematic review. Sensors, 16(1), p.18. 
SACHS, D. Sensor Fusion on Android Devices: A Revolution in Motion Processing . Google Tech talks. 2010. Disponível em https://www.youtube.com/watch?v=C7JQ7Rpwn2k

SEIFERT, K., \& CAMACHO, O. Implementing positioning algorithms using accelerometers. Freescale Semiconductor, 1-13. 2007. Disponível em http://persoetis.ensea.fr/ pierandr/cours/M1_SIC/AN3397.pdf

STAMM, A. \& THIEL, D.V., 2015. Investigating forward velocity and symmetry in freestyle swimming using inertial sensors. In Procedia Engineering. Elsevier Ltd, pp. $522-527$

STAMM, A., JAMES, D.A. \& THIEL, D.V., 2013. Velocity profiling using inertial sensors for freestyle swimming. Sports Engineering, 16(1), p.1-11.

SUNG, D. 2015. Best swimming wearables: Trackers, MP3 players and headphones. Wearable: tech for your connected self. Disponível em http://www.wareable.com/fitness-trackers/best-trackers-swimming-mp3 Acessado em junho de 2016.

WOODMAN, O. J. An introduction to inertial navigation. University of Cambridge, Computer Laboratory. 2007. Disponível http://www.cl.cam.ac.uk/techreports/UCAMCL-TR-696.pdf

XMETRICS. Sem ano. Disponível em http://www.xmetrics.it. Acessado em junho de 2016. 